\title{
Surface magnetism in iron, cobalt, and nickel
}

\section{Alde'n, M.; Mirbt, S.; Skriver, Hans Lomholt; Rosengaard, N. M.}

\section{Published in:}

Physical Review B

Link to article, DOI:

10.1103/PhysRevB.46.6303

Publication date:

1992

Document Version

Publisher's PDF, also known as Version of record

Link back to DTU Orbit

Citation (APA):

Alde'n, M., Mirbt, S., Skriver, H. L., \& Rosengaard, N. M. (1992). Surface magnetism in iron, cobalt, and nickel. Physical Review B, 46(10), 6303-6312. https://doi.org/10.1103/PhysRevB.46.6303

\section{General rights}

Copyright and moral rights for the publications made accessible in the public portal are retained by the authors and/or other copyright owners and it is a condition of accessing publications that users recognise and abide by the legal requirements associated with these rights.

- Users may download and print one copy of any publication from the public portal for the purpose of private study or research.

- You may not further distribute the material or use it for any profit-making activity or commercial gain

- You may freely distribute the URL identifying the publication in the public portal

If you believe that this document breaches copyright please contact us providing details, and we will remove access to the work immediately and investigate your claim. 


\title{
Surface magnetism in iron, cobalt, and nickel
}

\author{
M. Aldén and S. Mirbt \\ Department of Physics, University of Uppsala, Box 530, Uppsala, Sweden \\ H. L. Skriver and N. M. Rosengaard \\ Laboratory of Applied Physics, Technical University of Denmark, DK-2800 Lyngby, Denmark \\ B. Johansson \\ Department of Physics, University of Uppsala, Box 530, Uppsala, Sweden
}

(Received 25 March 1992)

\begin{abstract}
We have calculated magnetic moments, work functions, and surface energies for several of the most closely packed surfaces of iron, cobalt, and nickel by means of a spin-polarized Green's-function technique based on the linear muffin-tin orbitals method within the tight-binding and atomic sphere approximations. We find enhanced spin moments at all the surfaces considered except for Ni fcc(111), where the moment at the surface reverts to its bulk value. This is in close agreement with earlier slab calculations. In addition, we find that the calculated work functions and surface energies agree with experimental values to within $10 \%$, which may be considered most satisfactory in view of the computational efficiency of the Green's function technique. Exchange and correlation have been treated within the local spin-density approximation and we have considered three different parametrizations of the original many-body data. We find that the calculated work functions depend as much on the choice of this parametrization as on the effect of spin polarization.
\end{abstract}

\section{INTRODUCTION}

It is now well established from first-principles selfconsistent local spin-density calculations that the magnetic moment in the topmost layer(s) of magnetic $3 d-$ transition metals is generally enhanced with respect to the bulk magnetic moment. ${ }^{1-3}$ This is commonly explained as due to the reduction in coordination number and symmetry at the surface, which causes the $d$ bands to narrow and hence, in general, enhances the paramagnetic state density at the Fermi level $E_{F}$. The moment enhancement at the surface is particularly pronounced for $\mathrm{Fe} \mathrm{bcc(001),} \mathrm{where} \mathrm{the} \mathrm{majority} \mathrm{spin} \mathrm{band} \mathrm{is} \mathrm{not} \mathrm{al-}$ ready saturated in the bulk. This is in contrast to Co and $\mathrm{Ni}$, where the majority band is close to being saturated, thereby reducing the moment enhancement. ${ }^{4}$

The instability towards magnetic-moment formation in bulk systems is well accounted for by the Stoner theory of itinerant magnetism. Although the surface paramagnetic state density may be used to form a Stoner product ${ }^{1,5}$ and subsequently within a rigid-band model $^{6}$ used to explain the magnitude of the moment enhancement at a surface, no theory in terms of, e.g., the coordination number has been developed as yet. However, early findings of either enhanced or depressed magnetism at the surface and magnetically alive monolayers on top of bulk systems lead to discussions in terms of $d$-band narrowing and enhancement of the state density at $E_{F}$. Antiferromagnetic chromium is now known, by angle-resolved photoelectron spectroscopy ${ }^{7}$ and by full potential electronicstructure calculations, ${ }^{8}$ to exhibit ferromagnetic order at its bcc(001) surface. The surface magnetism of nickel was initially controversial until it was established that the surface magnetic moment is comparable to that of the bulk. ${ }^{3,9,10}$

The majority of the calculations of surface magnetism in the $3 d$ metals have been performed within a geometry consisting of either a single slab ${ }^{2,12-15}$ or of separated slabs in a three-dimensional supercell. While these slab methods have well-known deficiencies, the effect of which is not completely controlled, they will render accurate results when carried to convergence. ${ }^{3,6}$

By construction the Green's-function approach takes proper account of the broken symmetry at the surface, and hence does not suffer from the deficiencies of the slab methods. In addition, the Green's function approach is efficient in terms of computer time, and it may be carried to the same level of sophistication as the slab methods in terms of full potential and all-electron calculations, if needed. Several surface Green's function techniques have been presented in the near past. ${ }^{16-20}$ Feibelman $^{17}$ and Scheffler et al. ${ }^{20}$ have treated an impurity on a film and a surface, respectively, while results for perfect crystal surfaces have been reported by Inglesfield ${ }^{18}$ and Skriver and Rosengaard. ${ }^{19}$ Finally, Crampin et al. ${ }^{21}$ reported calculations of stacking faults energies by the related layer Korringa-Kohn-Rostoker (KKR) method.

In this paper we report on the implementation of a spin-polarized version of our Green's-function technique ${ }^{19}$ and present calculations of work functions, surface energies, and magnetic moments for several closepacked surfaces of ferromagnetic iron, cobalt, and nickel. 
Recently, Zeller et al. ${ }^{23}$ have used a related KKR Green'sfunction method to calculate the magnetic properties of single layers of iron, cobalt, and nickel on a copper surface.

In the test of our Green's-function method ${ }^{19}$ on the alkali metals we found that the calculated surface properties were quite sensitive to the choice of exchangecorrelation functional. In the present case, we therefore compare results obtained by the functional devised by von Barth and Hedin, ${ }^{24}$ which in the past has been extensively used in calculations for bulk magnetic systems, and the more recent one by Ceperley and Alder. ${ }^{25}$ For the latter we tested both the parametrization by Perdew and Zunger $^{27}$ in conjunction with the spin scaling of the correlation energy and potential as given by von Barth and Hedin $^{24}$ and that by Vosko, Wilk, and Nusair. ${ }^{26}$ The sensitivity to the spin interpolation was investigated for bulk iron by McLaren, Clougherty, and Albers, ${ }^{28}$ who found that only the Vosko-Wilk-Nusair functional yielded a bcc ferromagnetic ground state.

\section{COMPUTATIONAL METHOD}

The tight-binding (TB) linear-muffin-tin-orbitals (LMTO) Green's-function technique as implemented by Skriver and Rosengaard ${ }^{19}$ is based on the work by Andersen and co-workers. ${ }^{29-35}$ An essential aspect is the ability, within the atomic sphere approximation (ASA) and in the tight-binding representation, to generate the Green's-function matrices for a real, two-dimensional interface by a simple and efficient procedure. Since the details of the technique may be found in Ref. 19 we shall here restrict ourselves to an outline of the major steps with special emphasis on the spin dependence.

In a layer representation the Green's-function matrix may be expressed as

$$
G_{R_{\perp}^{\prime} L^{\prime}, R_{\perp} L ; \sigma}\left(\mathbf{k}_{\|}, z\right)=\frac{d_{\perp}}{2 \pi} e^{-i \mathbf{k}_{\|} \cdot\left(\mathbf{R}_{\|}-\mathbf{R}_{\|}^{\prime}\right)} \int_{0}^{2 \pi / d_{\perp}} d k_{\perp} e^{-i k_{\perp}\left(R_{\perp}-R_{\perp}^{\prime}\right)} G_{R^{\prime} L^{\prime}, R L ; \sigma}(\mathbf{k}, z),
$$

where $d_{\perp}$ is a layer distance, $\mathbf{k}=\left(\mathbf{k}_{\|}, k_{\perp}\right)$ is the decomposed reciprocal-space vector and $\mathbf{R}=\left(\mathbf{R}_{\|}, R_{\perp}\right)$ denotes sites in the three-dimensional (3D) primitive cell. The Green's-function matrix for the perfect crystal which enters (1) is obtained from

$$
G_{R^{\prime} L^{\prime}, R L ; \sigma}(\mathbf{k}, z)=\sum_{j} \frac{u_{R^{\prime} L^{\prime} ; \sigma}^{j}(\mathbf{k})\left[u_{R L ; \sigma}^{j}(\mathbf{k})\right]^{*}}{z-\epsilon_{j ; \sigma}(\mathbf{k})}
$$

where $u^{j}(\mathbf{k})$ are the eigenvectors and $\epsilon_{j}(\mathbf{k})$ the eigenvalues of the TB-LMTO Hamiltonian in the orthogonal $(\gamma)$ representation, ${ }^{32,34} L$ refers to the combined angularmomentum quantum numbers $(l, m)$, and $\sigma$ labels spin. To calculate the energy moments of the state density the Green's-function matrices are evaluated for complex energies $z$ on a semicircular contour, which encloses all the occupied states and cuts the real axis at $E_{F}$.

The Green's-function matrices for the two semiinfinite crystals are obtained by a Löwdin downfolding technique, ${ }^{36}$ which hinges on the fact that the LMTO structure constants in the most localized representation denoted by $\beta$ are short ranged, i.e.,

$$
S_{A B}^{\beta}=0 \text { for } \quad\{A B\} \neq\{a b\} .
$$

Here, $A B$ refers to the two half spaces separated by the surface and $\{a b\}$ is the small subset of layers where the hopping is nonzero. After the transformation ${ }^{19,32,34}$ of the layer Green's-function matrix (1) into the form defined by the KKR-ASA equations in the $\beta$ representation the Löwdin downfolded ideal Green's-function matrices for the bulk $(X)$ and the vacuum $(Y)$ are given by

$$
\tilde{g}_{A A ; \sigma}^{X}=g_{A A ; \sigma}^{X}-g_{A b ; \sigma}^{X}\left(g_{b b ; \sigma}^{X}\right)^{-1} g_{b A ; \sigma}^{X},
$$

$$
\tilde{g}_{B B}^{Y}=g_{B B}^{Y}-g_{B a}^{Y}\left(g_{a a}^{Y}\right)^{-1} g_{a B}^{Y},
$$

where the spin label has been omitted for the vacuum Green's function. The surface Green's-function matrices $g$ for the unrelaxed, combined system is found from the definition of the KKR-ASA Green's function written in the block form

$$
\left(\begin{array}{cc}
\left(\tilde{g}_{a a ; \sigma}^{X}\right)^{-1} & -S_{a b} \\
-S_{b a} & \left(\tilde{g}_{b b}^{Y}\right)^{-1}
\end{array}\right)\left(\begin{array}{cc}
g_{a a ; \sigma} & g_{a b ; \sigma} \\
g_{b a ; \sigma} & g_{b b ; \sigma}
\end{array}\right)=\left(\begin{array}{cc}
1_{a a} & 0_{a b} \\
0_{a b} & 1_{b b}
\end{array}\right)
$$

The effect of relaxing the potentials close to the surface is found from the finite Dyson equation,

$$
g_{\sigma}^{s}=g_{\sigma}+g_{\sigma} \Delta P_{\sigma}^{s} g_{\sigma}^{s}
$$

where the surface Green's-function matrix $g^{s}$ is expressed in terms of the unrelaxed Green's-function matrix and the diagonal potential-function matrix $\Delta P^{s}$, which describes the relaxation of the potentials.

The atom-centered, spherically symmetric ASA potentials $V_{R s ; \sigma}$ are obtained by solving the radial Poisson equation within the atomic spheres. To this we add the electrostatic monopole and dipole contributions from the neighboring spheres by the multipole expansion

$$
V_{R ; \sigma}\left(r_{R}\right)=V_{R s ; \sigma}\left(r_{R}\right) Y_{s}+\frac{1}{S} \sum_{R^{\prime} L^{\prime}} M_{R R^{\prime}}^{s L^{\prime}} Q_{R^{\prime}}^{L^{\prime}}
$$

where the Madelung matrices $M_{R R^{\prime}}^{s L^{\prime}}$ are given by Skriver and Rosengaard ${ }^{19}$ and the multipole moments $Q_{R^{\prime}}^{L^{\prime}}$ are obtained by contour integration of the surface Green's function $g^{s}$.

The total energy in the ASA may be expressed as the sum of the Madelung energy, the kinetic energy, the electrostatic energy, and the exchange-correlation energy in the form 


$$
\begin{aligned}
E= & \sum_{R L, R^{\prime} L^{\prime}} Q_{R}^{L} M_{R R^{\prime}}^{L L^{\prime}} Q_{R^{\prime}}^{L^{\prime}}+\sum_{R ; \sigma} T_{R ; \sigma}^{v} \\
& -\frac{1}{\sqrt{4 \pi}} \sum_{R ; \sigma} \int_{R} d^{3} r n_{R s ; \sigma}^{v}(r) \frac{2 Z_{R}}{r}+\frac{1}{\sqrt{4 \pi}} \sum_{R ; \sigma} \int_{R} d^{3} r n_{R s ; \sigma}^{v}(r)\left(\frac{1}{2} V_{R s ; \sigma}^{v}(r)+V_{R s ; \sigma}^{c}(r)\right) \\
& +\frac{1}{\sqrt{4 \pi}} \sum_{R ; \sigma} \int_{R} d^{3} r n_{R s ; \sigma}(r) \epsilon_{\mathrm{xc}}\left[n_{R s ; \uparrow}(r), n_{R s ; \downarrow}(r)\right]-\frac{1}{\sqrt{4 \pi}} \sum_{R ; \sigma} \int_{R} d^{3} r n_{R s ; \sigma}^{c} \epsilon_{\mathrm{xc}}\left[n_{R s}^{c}(r)\right],
\end{aligned}
$$

where the atomic-sphere projected kinetic energy may be obtained from the sum of the one-electron energies and the effective potential as

$$
\begin{aligned}
T_{R ; \sigma}^{v}= & \frac{1}{2 \pi i} \oint d z z G_{R L^{\prime}, R L^{\prime \prime}}^{\gamma}(z) \\
& -\frac{1}{\sqrt{4 \pi}} \int_{R} d^{3} r n_{R s ; \sigma}^{v}(r) V_{R ; \sigma}(r) .
\end{aligned}
$$

In Eqs. (9) and (10) the charges and the electrostatic potentials are separated into valence and core contributions, indicated by the superscripts $v$ and $c$, respectively, terms pertaining to the (frozen) core are neglected, and $\epsilon_{\mathbf{x c}}$ is the parametrized exchange-correlation energy density in the local-spin-density approximation. Finally, the ASA surface energy is evaluated as the difference between (9) for the surface layers and the corresponding expression for the perfect crystal.

In the present calculations we used $16(\mathrm{Fe}, \mathrm{Co})$ and 24 (Ni) $z$ points logarithmically spaced on a semicircular contour in the lower half of the complex energy plane. The larger number for $\mathrm{Ni}$ was chosen to give an adquate representation of its sharp $d$ state density close to the Fermi level. In a related study Inglesfield and Benesh, ${ }^{18}$ used 63 sampling points for $\mathrm{Ni}$.

The number of $\mathbf{k}_{\|}$points were taken to be 36,36 , 45,45 , and 64 special points ${ }^{37}$ in the irreducible part of the 2D Brillouin zone for $\mathrm{bcc}(001)$, fcc(001), fcc(111), $h c p(001)$, and bcc(110), respectively, in addition to the $50 k_{\perp}$ points used to calculate the layer Green's-function matrix (1). The same choices of $\mathbf{k}$ points were used in the underlying bulk calculations thus leading to an effective cancellation of errors in the total-energy differences.

In the calculations we used $s, p$, and $d$ muffin-tin orbitals and included 3-5 metal surface layers and 2 vacuum layers depending on convergence tests. The frozencore contributions for $\mathrm{Fe}, \mathrm{Co}$, and $\mathrm{Ni}$ were calculated for the free atoms with the valence configurations $3 d^{7} 4 s^{1}$, $3 d^{7} 4 s^{2}$, and $3 d^{8} 4 s^{2}$, respectively. A $3 d^{6} 4 s^{2}$ atomic configuration for $\mathrm{Fe}$ was tested for $\mathrm{Fe}(001)$ and was found to affect the calculated surface magnetic moment and work function by less than $1 \%$ and the surface energy by less than $2 \%$.

\section{RESULTS}

Our results for layer- and orbital-decomposed total charges and magnetic moments together with calculated work functions and surface energies for seven $3 d$ surfaces are presented in Tables I-VII. The values listed are those obtained by means of the Vosko-Wilk-Nusair ${ }^{26}$ parametrization of the Ceperley-Alder (CA) exchangecorrelation functional. ${ }^{25}$ In the tables we also quote results from earlier work and list the value of the WignerSeitz radius and the number of layers used in the calculations. Finally, the calculated work functions and surface energies are shown in Figs. 1 and 2 as functions of atomic number, surface facet, and exchange-correlation potential.

It follows from Fig. 1 that the calculated work function is sensitive to the choice of exchange-correlation potential, and that the effect is as large as $0.4 \mathrm{eV}$, i.e., comparable to the effect of the spin polarization itself. This shift in the work function obtained by the von BarthHedin $^{24}$ parametrization on the one hand and by the two CA parametrizations on the other is a direct re-

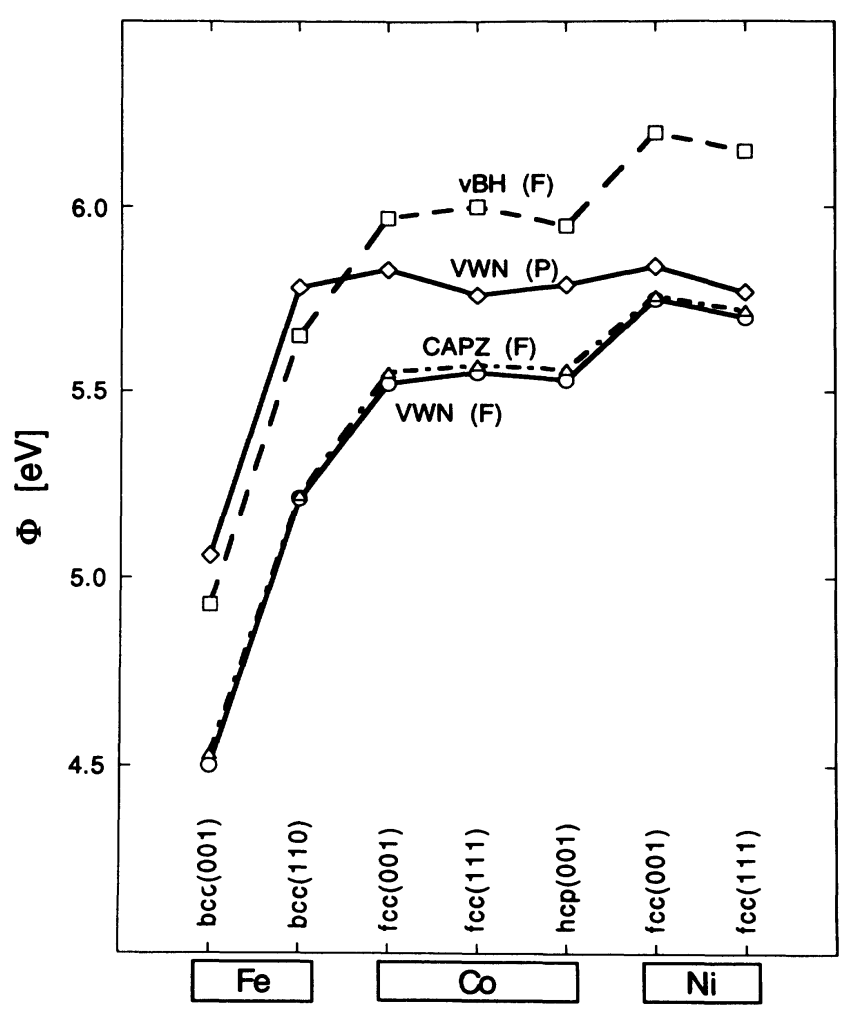

FIG. 1. Calculated work functions for all the surfaces considered. The results are obtained by means of different parametrizations of the exchange-correlation potential as explained in the text, and include spin-polarized $(F)$ as well as paramagnetic $(P)$ calculations. 


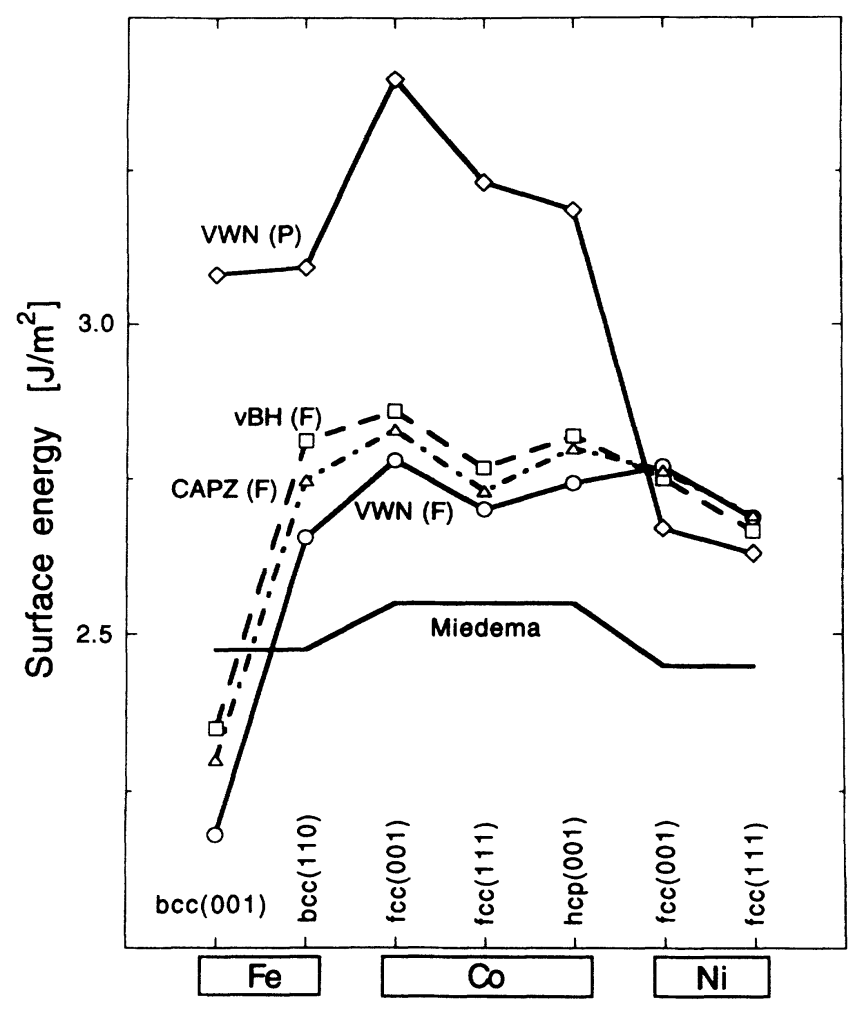

FIG. 2. Calculated surface energies obtained within the ASA for various exchange-correlation potentials (symbols as in text). The paramagnetic and spin-polarized calculation are denoted by $P$ and $F$, respectively. Values derived from surface tensions (Refs. 40 and 41) are labeled as Miedema.

sult of the systematic difference between the exchangecorrelation potentials themselves, as may be seen from Fig. 3. We note that the Ceperley-Alder-type potentials give the best agreement with the experimental work functions compiled by Michaelson. ${ }^{39}$

The calculated surface energies presented in Fig. 2 are found to be less affected by the choice of exchangecorrelation potential than the work functions, while the effect of spin polarization is found to be appreciable for $\mathrm{Fe}$ and $\mathrm{Co}$. In the comparison with the surface energies recommended by de Boer et $a l^{40}$ one should note that their values are derived from surface tensions at the melting temperature and hence reflect average values independent of surface facets. We find that also for the surface energy the Ceperley-Alder-type potential gives the best agreement with values derived from experiment.

\section{A. Iron; bcc(110) and bcc(001)}

In the calculations for the bcc(110) surface of iron we have treated three metal layers and two vacuum layers $(3+2)$ self-consistently. As may be seen from Table I, we find no Friedel-type oscillations in the magnetic moments, and we find a surface magnetic moment of 2.57 $\mu_{B}$, which is an increase of $0.3 \mu_{B}$, or $14 \%$, over the bulk value. This increase is somewhat lower than the $20 \%$

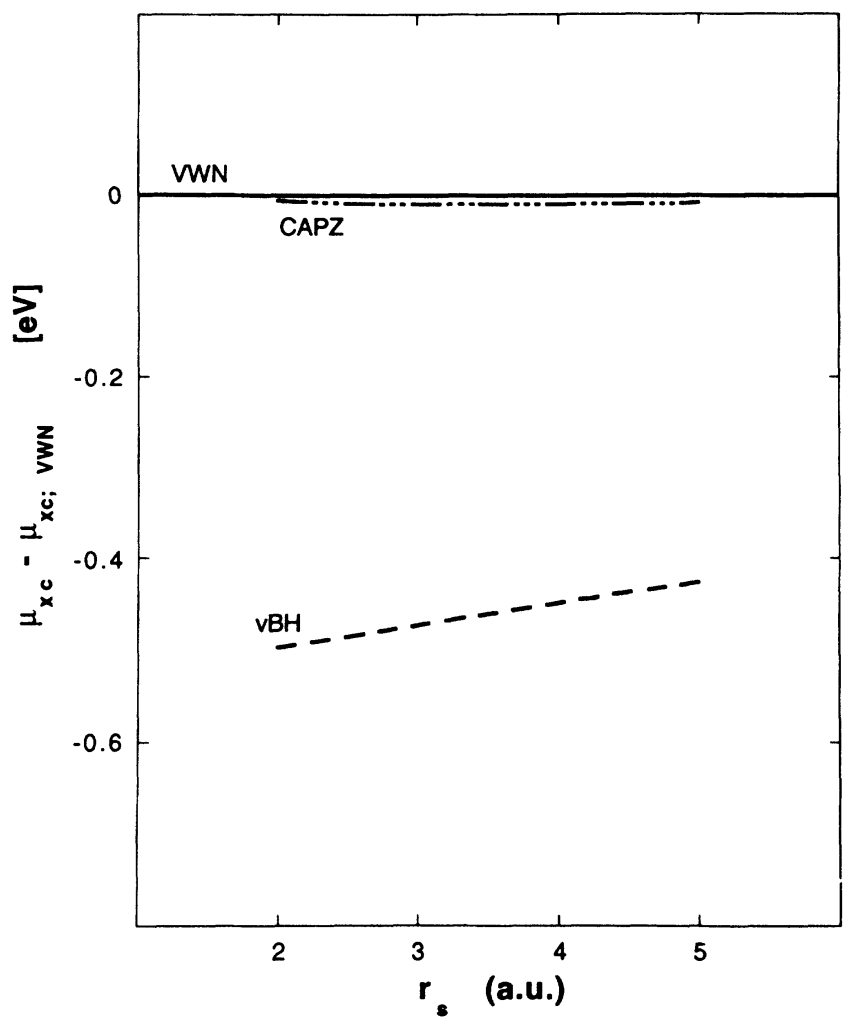

FIG. 3. The difference in the magnitude of the three exchange-correlation potentials used in the calculations. Symbols are as in text.

found in the full-potential linearized augmented plane wave (FLAPW) calculation by Fu and Freeman ${ }^{42}$ and the $30 \%$ found in a recent spin-polarized low-energy electron diffraction experiment. ${ }^{11}$ Our calculated work function is $5.21 \mathrm{eV}$, a value slightly larger than those reported from various experiments on $\mathrm{Fe}$, although no measurements of the work function for the (110) facet has been reported to our knowledge. The surface energy is calculated within the ASA to be $2.66 \mathrm{~J} / \mathrm{m}^{2}$, which is within $10 \%$ of the value estimated by de Boer et al..$^{40}$

Table II shows the magnetic moment and charge density of the more open bcc(001) surface, for which we have treated $(5+2)$ layers. This surface is the most loosely packed of the surfaces considered here, and the electronic structure differs somewhat from the other systems. A noticeably large amount of charge is lost from the metal surface layer $(S)$, i.e., 0.50 electrons, out of which 0.46 electrons, originally of mainly $p$ character, are transferred into the $(S+1)$ layer forming surface $s$ and $p$ states. The calculated surface energy for the paramagnetic case is equal to that of the paramagnetic bcc(110) facet, 3.1 $\mathrm{J} / \mathrm{m}^{2}$. When allowing for spin polarization, the large gain in exchange energy at the (001) surface lowers the surface energy by as much as $0.46 \mathrm{eV} /$ atom, becoming only $2.18 \mathrm{~J} / \mathrm{m}^{2}$. Our calculated work function is 4.50 $\mathrm{eV}$ lying well within the scatter of the experimental results $(4.4-4.8 \mathrm{eV})$. We find a surface magnetic moment of $2.97 \mu_{B}$, which is in very good agreement with earlier FLAPW and full-potential linear muffin-tin orbital 
TABLE I. Fe bcc(110).

\begin{tabular}{|c|c|c|c|c|c|}
\hline \multirow{2}{*}{$\begin{array}{c}\mathrm{r}_{W S}=2.662 \text { (a.u.) } \\
(3+2) \text { layers }\end{array}$} & \multirow[b]{2}{*}{$s$} & \multicolumn{3}{|c|}{ TB LMTO (ASA) } & \multirow{2}{*}{$\begin{array}{l}\text { Other calc., expt. } \\
\text { Total in layer }\end{array}$} \\
\hline & & $p$ & $d$ & Total in layer & \\
\hline \multicolumn{6}{|c|}{ Charges } \\
\hline$S+1$ & 0.13 & 0.09 & 0.03 & 0.26 & \\
\hline$S$ & 0.65 & 0.57 & 6.54 & 7.75 & \\
\hline$S-1$ & 0.66 & 0.79 & 6.54 & 7.99 & \\
\hline$S-2$ & 0.67 & 0.80 & 6.53 & 8.00 & \\
\hline \multicolumn{6}{|c|}{ Magnetic moments } \\
\hline$S+1$ & 0.00 & 0.01 & 0.01 & 0.01 & \\
\hline$S$ & 0.00 & 0.01 & 2.57 & 2.57 & $2.65^{\mathrm{a}}$ \\
\hline$S-1$ & -0.01 & -0.05 & 2.41 & 2.35 & $2.37^{\mathrm{a}}$ \\
\hline$S-2$ & -0.01 & -0.06 & 2.32 & 2.25 & $2.25^{\mathrm{a}}$ \\
\hline Work function (eV) & \multicolumn{4}{|c|}{5.21} & \\
\hline Surface energy $\left(\mathrm{J} / \mathrm{m}^{2}\right)$ & \multicolumn{4}{|c|}{2.66} & $2.48^{\mathrm{b}}$ \\
\hline
\end{tabular}

${ }^{a}$ FLAPW, seven-layer slab (Ref. 42).

${ }^{b}$ Experiment (surface independent) (Ref. 40).

(FLMTO) calculations. ${ }^{4,6}$ However, in contrast to the results of the latter calculations, our layer-decomposed magnetic moments converge towards the bulk value away from the surface, as a result of the (correct) boundary condition fulfilled by the Green's function. This is illustrated in Fig. 4, showing also the small Friedel oscillations appearing in the layer-decomposed magnetic mo- ments. It is interesting to note that these oscillations are of exactly the same character and size as those found in the FLAPW calculation by Oshnishi and Freeman. ${ }^{6}$ They are, however, not apparent in the recent work by Eriksson et al., ${ }^{4}$ where spin-orbit coupling was included in the FLMTO Hamiltonian together with so-called orbital polarization.

TABLE II. Fe bcc(001).

\begin{tabular}{|c|c|c|c|c|c|}
\hline \multirow{2}{*}{$\begin{array}{c}\left.\mathrm{r}_{W S}=2.662 \text { (a.u. }\right) \\
(5+2) \text { layers }\end{array}$} & \multirow[b]{2}{*}{$s$} & \multicolumn{2}{|c|}{ TB LMTO (ASA) } & \multirow[b]{2}{*}{ Total in layer } & \multirow{2}{*}{$\begin{array}{l}\text { Other calc., expt } \\
\text { Total in layer }\end{array}$} \\
\hline & & $p$ & $d$ & & \\
\hline \multicolumn{6}{|c|}{ Charges } \\
\hline$S+1$ & 0.27 & 0.14 & 0.06 & 0.46 & \\
\hline$S$ & 0.60 & 0.46 & 6.43 & 7.49 & $6.78^{a}$ \\
\hline$S-1$ & 0.67 & 0.78 & 6.57 & 8.02 & $7.05^{\mathrm{a}}$ \\
\hline$S-2$ & 0.67 & 0.80 & 6.52 & 7.99 & $7.05^{\mathrm{a}}$ \\
\hline$S-3$ & 0.67 & 0.80 & 6.53 & 8.00 & $7.05^{\mathrm{a}}$ \\
\hline$S-4$ & 0.67 & 0.80 & 6.53 & 8.00 & \\
\hline \multicolumn{6}{|c|}{ Magnetic moments } \\
\hline$S+1$ & 0.00 & 0.03 & 0.02 & 0.04 & \\
\hline$S$ & 0.01 & 0.02 & 2.94 & 2.97 & $2.98,^{\mathrm{a}} 2.87^{\mathrm{b}}$ \\
\hline$S-1$ & -0.01 & -0.04 & 2.35 & 2.30 & $2.35,2.34^{\mathrm{b}}$ \\
\hline$S-2$ & 0.00 & -0.05 & 2.43 & 2.37 & $2.39,^{\mathrm{a}} 2.33^{\mathrm{b}}$ \\
\hline$S-3$ & -0.01 & -0.06 & 2.31 & 2.25 & $2.25,^{\mathrm{a}} 2.18^{\mathrm{b}}$ \\
\hline$S-4$ & -0.01 & -0.06 & 2.31 & 2.24 & \\
\hline Work function (eV) & \multicolumn{4}{|c|}{4.50} & $4.29,^{\mathrm{a}} 4.4^{\mathrm{c}}$ \\
\hline Surface energy $\left(\mathrm{J} / \mathrm{m}^{2}\right)$ & \multicolumn{4}{|c|}{2.18} & $2.48^{\mathrm{d}}$ \\
\hline
\end{tabular}

${ }^{a}$ FLAPW, seven-layer slab (quoted charges are inside touching MT spheres) (Ref. 6).

${ }^{b}$ FLMTO spin-orbit, seven-layer slab (spin moments) (Ref. 4).

${ }^{\mathrm{c}}$ Experiment (Ref. 38).

${ }^{d}$ Experiment (surface independent) (Ref. 40). 


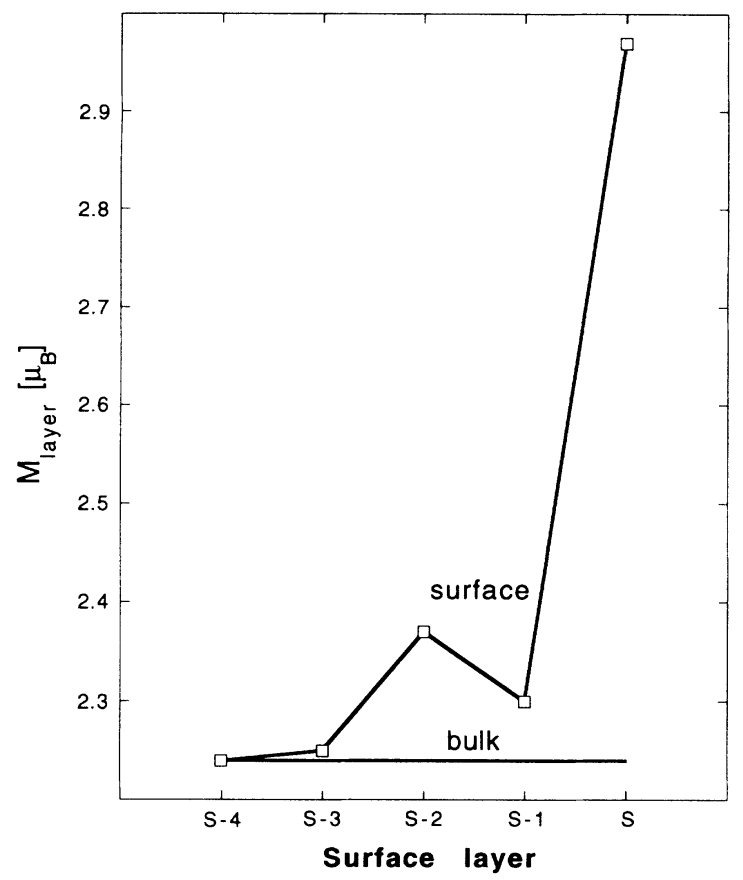

FIG. 4. Layer-decomposed total magnetic moments of $\mathrm{Fe}(001)$, compared to the value in the bulk.

B. Cobalt; hcp(001), fcc(001), and fcc(111)

Cobalt crystallizes in the hcp structure below $\sim 950 \mathrm{~K}$ but the fcc phase may be stabilized experimentally at room temperature by rapid quenching. ${ }^{43}$ We have therefore performed calculations for cobalt both in the fcc and the hcp crystal structures, permitting us to compare our results with earlier calculations. For the fcc(001) surface we obtain a surface-layer magnetic moment of $1.84 \mu_{B}$, which is a $12 \%$ increase over the bulk value of $1.64 \mu_{B}$. This is in close agreement with the $13 \%$ increase obtained inside muffin-tin spheres by the FLAPW method. ${ }^{22}$ The moments in the subsurfaces are bulk-like, similiar to the FLAPW result, as can be seen from Table III.

Results for the more close-packed fcc(111) surface are shown in Table IV. Here the calculated enhancement in the magnetic moment at the surface is only $5.5 \%$, which may be explained as a result of the increased surface cordination number. The total moment is also somewhat quenched by the $p$ states. In this case the close-packed geometry of the surface layers seems to produce a more smooth enhancement of the magnetism towards the surface than was found for the fcc(001) surface. The results for the $h c p(001)$ surface are found to be very similar to those of the fcc(111). This is to be expected, since the packing of these two surfaces is very similar, apart from the scaling. The surface and subsurface magnetic moments are enhanced by $5.6 \%$ and $2 \%$, respectively, over the hcp bulk value of $1.61 \mu_{B}$ (see Table V).

The calculated work functions are 5.52, 5.55, and 5.53 $\mathrm{eV}$ for the $\mathrm{fcc}(001)$, fcc(111), and hcp(001) surfaces, respectively. These may be compared to the FLAPW result [fcc(001): $5.17 \mathrm{eV}$ ] and to experiment $(\sim 5.0 \mathrm{eV})$. Our ASA-calculated surface energies are $2.78,2.70$, and 2.74 $\mathrm{J} / \mathrm{m}^{2}$, respectively.

\section{Nickel; fcc(001) and fcc(111)}

Our results for the two most closely packed fcc surfaces support the general consensus that the nickel surface is

TABLE III. Co fcc(001).

\begin{tabular}{|c|c|c|c|c|c|}
\hline \multirow{2}{*}{$\begin{array}{c}\mathrm{r}_{W S}=2.617 \text { (a.u.) } \\
(4+2) \text { layers }\end{array}$} & \multirow[b]{2}{*}{$s$} & \multicolumn{2}{|c|}{ TB LMTO (ASA) } & \multirow[b]{2}{*}{ Total in layer } & \multirow{2}{*}{$\begin{array}{l}\text { Other calc., expt. } \\
\text { Total in layer }\end{array}$} \\
\hline & & $p$ & $d$ & & \\
\hline \multicolumn{6}{|c|}{ Charges } \\
\hline$S+1$ & 0.18 & 0.11 & 0.04 & 0.33 & \\
\hline$S$ & 0.64 & 0.52 & 7.50 & 8.66 & $8.04^{\mathrm{a}}$ \\
\hline$S-1$ & 0.68 & 0.80 & 7.53 & 9.01 & $8.27^{\mathrm{a}}$ \\
\hline$S-2$ & 0.68 & 0.81 & 7.51 & 9.00 & $8.27^{\mathrm{a}}$ \\
\hline$S-3$ & 0.68 & 0.81 & 7.51 & 9.00 & $8.27^{\mathrm{a}}$ \\
\hline \multicolumn{6}{|c|}{ Magnetic moments } \\
\hline$S+1$ & -0.02 & 0.00 & 0.00 & -0.01 & \\
\hline$S$ & 0.00 & -0.01 & 1.85 & 1.84 & $1.86^{\mathrm{a}}$ \\
\hline$S-1$ & -0.01 & -0.05 & 1.69 & 1.63 & $1.64^{\mathrm{a}}$ \\
\hline$S-2$ & -0.01 & -0.06 & 1.73 & 1.66 & $1.65^{\mathrm{a}}$ \\
\hline$S-3$ & -0.01 & -0.06 & 1.71 & 1.64 & $1.64^{\mathrm{a}}$ \\
\hline Work function $(\mathrm{eV})$ & \multicolumn{4}{|c|}{5.52} & $5.17,^{\mathrm{a}} 5.0^{\mathrm{b}}$ \\
\hline Surface energy $\left(\mathrm{J} / \mathrm{m}^{2}\right)$ & \multicolumn{4}{|c|}{2.78} & $2.55^{\mathrm{c}}$ \\
\hline
\end{tabular}

${ }^{a}$ FLAPW, nine-layer slab (charges and moments inside touching MT spheres) (Ref. 22).

${ }^{\mathrm{b}}$ Experiment (Ref. 39).

${ }^{\mathrm{c}}$ Experiment (surface independent) (Ref. 40). 
TABLE IV. Co fcc(111).

\begin{tabular}{|c|c|c|c|c|c|}
\hline \multirow{2}{*}{$\begin{array}{c}\mathrm{r}_{W S}=2.617 \text { (a.u.) } \\
(3+2) \text { layers }\end{array}$} & \multirow[b]{2}{*}{$s$} & \multicolumn{2}{|c|}{ TB LMTO (ASA) } & \multirow[b]{2}{*}{ Total in layer } & \multirow{2}{*}{$\begin{array}{l}\text { Other calc., expt. } \\
\text { Total in layer }\end{array}$} \\
\hline & & $p$ & $d$ & & \\
\hline \multicolumn{6}{|c|}{ Charges } \\
\hline$S+1$ & 0.12 & 0.08 & 0.03 & 0.24 & \\
\hline$S$ & 0.66 & 0.61 & 7.51 & 8.77 & \\
\hline$S-1$ & 0.68 & 0.80 & 7.52 & 8.99 & \\
\hline$S-2$ & 0.68 & 0.80 & 7.51 & 9.00 & \\
\hline \multicolumn{6}{|c|}{ Magnetic moments } \\
\hline$S+1$ & -0.01 & 0.00 & 0.00 & -0.01 & \\
\hline$S$ & -0.01 & -0.04 & 1.77 & 1.72 & \\
\hline$S-1$ & -0.01 & -0.06 & 1.74 & 1.67 & \\
\hline$S-2$ & -0.01 & -0.06 & 1.70 & 1.63 & \\
\hline Work function $(\mathrm{eV})$ & \multicolumn{4}{|c|}{5.55} & $5.0^{\mathrm{a}}$ \\
\hline Surface energy $\left(\mathrm{J} / \mathrm{m}^{2}\right)$ & \multicolumn{4}{|c|}{2.70} & $2.55^{\mathrm{b}}$ \\
\hline
\end{tabular}

${ }^{a}$ Experiment (Ref. 39).

${ }^{\mathrm{b}}$ Experiment (surface independent) (Ref. 40).

not magnetically dead but carries a magnetic moment comparable to that of the bulk. At the fcc(001) surface the calculated moment is $0.69 \mu_{B}$, which is a slight increase over our bulk value of $0.64 \mu_{B}$ (Table VI). Similarly to the case of $\mathrm{Co} f \mathrm{cc}(001)$, the orbital composition of the subsurfaces appears very bulklike with only a minor Friedel oscillation in the magnetic moment. In earlier full-potential slab calculations for this surface these details differ somewhat as regards the layer behavior. The FLMTO spin-orbit calculations ${ }^{4}$ gave an enhancement similar to ours, but the magnetic moment was found to increase more smoothly through the layers. The FLAPW study $^{3}$ yielded a slightly larger enhancement (20\%), and the variation in the magnetic moment with layers was found to be intermediate between our results and those of the FLMTO calculation. In the case of the fcc(111) surface we found, see Table VII, that the moment increased continuously through the inner layers similarly to the case of Co fcc(111), but reverted to its bulk value at the surface layer. This type of halted increase of the surface magnetic moment was also found in a FLAPW study on the $\mathrm{Ni}(111)$ surface by $\mathrm{Fu}$ and Freeman. ${ }^{44}$

TABLE V. Co hcp(001).

\begin{tabular}{|c|c|c|c|c|c|}
\hline \multirow{2}{*}{$\begin{array}{c}\mathrm{r}_{W S}=2.621 \text { (a.u.) } \\
(4+2) \text { layers }\end{array}$} & \multirow[b]{2}{*}{$s$} & \multicolumn{3}{|c|}{ TB LMTO (ASA) } & \multirow{2}{*}{$\begin{array}{l}\text { Other calc., expt. } \\
\text { Total in layer }\end{array}$} \\
\hline & & $p$ & $d$ & Total in layer & \\
\hline \multicolumn{6}{|c|}{ Charges } \\
\hline$S+1$ & 0.12 & 0.08 & 0.03 & 0.24 & \\
\hline$S$ & 0.66 & 0.60 & 7.51 & 8.77 & \\
\hline$S-1$ & 0.68 & 0.79 & 7.52 & 8.99 & \\
\hline$S-2$ & 0.68 & 0.80 & 7.52 & 9.00 & \\
\hline$S-3$ & 0.68 & 0.80 & 7.52 & 9.00 & \\
\hline \multicolumn{6}{|c|}{ Magnetic moments } \\
\hline$S+1$ & -0.01 & 0.00 & 0.00 & -0.01 & \\
\hline$S$ & -0.01 & -0.05 & 1.76 & 1.70 & $1.75^{\mathrm{a}}$ \\
\hline$S-1$ & -0.01 & -0.06 & 1.72 & 1.65 & $1.67^{\mathrm{a}}$ \\
\hline$S-2$ & -0.01 & -0.07 & 1.68 & 1.60 & $1.58^{\mathrm{a}}$ \\
\hline$S-3$ & -0.01 & -0.07 & 1.69 & 1.61 & \\
\hline Work function $(\mathrm{eV})$ & \multicolumn{4}{|c|}{5.53} & $5.0^{\mathrm{b}}$ \\
\hline Surface energy $\left(\mathrm{J} / \mathrm{m}^{2}\right)$ & \multicolumn{4}{|c|}{2.74} & $2.55^{\mathrm{c}}$ \\
\hline
\end{tabular}

${ }^{a}$ FLMTO spin-orbit, five-layer slab (spin moments) (Ref. 4).

${ }^{b}$ Experiment (Ref. 39).

${ }^{\mathrm{c}}$ Experiment (surface independent) (Ref. 40). 
TABLE VI. Ni fcc(001).

\begin{tabular}{|c|c|c|c|c|c|}
\hline \multirow{2}{*}{$\begin{array}{c}\mathrm{r}_{W S}=2.598 \text { (a.u.) } \\
(4+2) \text { layers }\end{array}$} & \multirow[b]{2}{*}{$s$} & \multicolumn{2}{|c|}{ TB LMTO (ASA) } & \multirow[b]{2}{*}{ Total in layer } & \multirow{2}{*}{$\begin{array}{l}\text { Other calc., expt. } \\
\text { Total in layer }\end{array}$} \\
\hline & & $p$ & $d$ & & \\
\hline \multicolumn{6}{|c|}{ Charges } \\
\hline$S+1$ & 0.16 & 0.11 & 0.04 & 0.31 & $0.14^{\mathrm{a}}$ \\
\hline$S$ & 0.64 & 0.51 & 8.55 & 9.69 & $9.86^{\mathrm{a}}$ \\
\hline$S-1$ & 0.68 & 0.77 & 8.55 & 10.00 & $10.00^{\mathrm{a}}$ \\
\hline$S-2$ & 0.69 & 0.78 & 8.53 & 10.00 & $10.00^{\mathrm{a}}$ \\
\hline$S-3$ & 0.69 & 0.77 & 8.54 & 10.00 & $10.00^{\mathrm{a}}$ \\
\hline \multicolumn{6}{|c|}{ Magnetic moments } \\
\hline$S+1$ & -0.01 & 0.00 & 0.00 & -0.01 & $-0.005^{\mathrm{a}}$ \\
\hline$S$ & 0.00 & -0.02 & 0.71 & 0.69 & $0.68,^{\mathrm{a}} \quad 0.59^{\mathrm{b}}$ \\
\hline$S-1$ & 0.00 & -0.03 & 0.67 & 0.64 & $0.60,^{\mathrm{a}} \quad 0.58^{\mathrm{b}}$ \\
\hline$S-2$ & 0.00 & -0.03 & 0.69 & 0.66 & $0.59,^{\mathrm{a}} \quad 0.57^{\mathrm{b}}$ \\
\hline$S-3$ & 0.00 & -0.03 & 0.67 & 0.64 & $0.56,^{\mathrm{a}} \quad 0.55^{\mathrm{b}}$ \\
\hline Work function (eV) & \multicolumn{4}{|c|}{5.75} & $5.22,^{\mathrm{c}} \quad 5.37,^{\mathrm{a}} 5.71^{\mathrm{d}}$ \\
\hline Surface energy $\left(\mathrm{J} / \mathrm{m}^{2}\right)$ & \multicolumn{4}{|c|}{2.77} & $2.45^{\mathrm{e}}$ \\
\hline
\end{tabular}

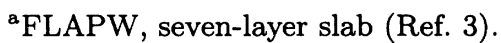

${ }^{b}$ FLMTO spin-orbit, seven-layer slab (spin-moments) (Ref. 4).

${ }^{\mathrm{c}}$ Experiment (Ref. 39).

${ }^{\mathrm{d} F u l l-p o t e n t i a l}$ Green's-function method (paramagnetic) (Ref. 18).

${ }^{\mathrm{e}}$ Experiment (surface independent) (Ref. 40).

Our calculated surface energies are $2.77 \mathrm{~J} / \mathrm{m}^{2}$ and 2.69 $\mathrm{J} / \mathrm{m}^{2}$ for the $\mathrm{fcc}(001)$ and $\mathrm{fcc}(111)$ surfaces, respectively, and our calculated work functions are $5.75 \mathrm{eV}$ and 5.70 $\mathrm{eV}$, respectively. The trends found here are analogous to those exhibited by cobalt. First, the increased total charge redistribution when going from fcc(111) to fcc(001) increases the surface energy. Secondly, our cal- culated work functions are slightly larger than both the experimental results ( $5.22 \mathrm{eV}$ and $5.35 \mathrm{eV}$, respectively) and the $5.37 \mathrm{eV}$ obtained in the FLAPW calcultion for the $\mathrm{Ni}(001)$ surface. On the other hand our calculated work functions are close to the value of $5.71 \mathrm{eV}$ reported in a recent full-potential Green's-function calculation for paramagnetic fcc $(001) \mathrm{Ni}^{18}$

TABLE VII. Ni fcc(111).

\begin{tabular}{|c|c|c|c|c|c|}
\hline \multirow{2}{*}{$\begin{array}{c}\mathrm{r}_{W S}=2.598 \text { (a.u.) } \\
\quad(4+2) \text { layers }\end{array}$} & \multirow[b]{2}{*}{$s$} & \multicolumn{2}{|c|}{ TB LMTO (ASA) } & \multirow[b]{2}{*}{ Total in layer } & \multirow{2}{*}{$\begin{array}{l}\text { Other calc., expt. } \\
\text { Total in layer }\end{array}$} \\
\hline & & $p$ & $d$ & & \\
\hline \multicolumn{6}{|c|}{ Charges } \\
\hline$S+1$ & 0.11 & 0.08 & 0.03 & 0.22 & \\
\hline$S$ & 0.66 & 0.58 & 8.54 & 9.79 & \\
\hline$S-1$ & 0.68 & 0.77 & 8.54 & 9.99 & \\
\hline$S-2$ & 0.69 & 0.77 & 8.54 & 10.00 & \\
\hline$S-3$ & 0.69 & 0.78 & 8.54 & 10.00 & \\
\hline \multicolumn{6}{|c|}{ Magnetic moments } \\
\hline$S+1$ & -0.01 & 0.00 & 0.00 & -0.01 & \\
\hline$S$ & 0.00 & -0.03 & 0.65 & 0.62 & $0.63^{a}$ \\
\hline$S-1$ & 0.00 & -0.03 & 0.71 & 0.67 & $0.64^{\mathrm{a}}$ \\
\hline$S-2$ & 0.00 & -0.03 & 0.68 & 0.65 & $0.58^{\mathrm{a}}$ \\
\hline$S-3$ & 0.00 & -0.03 & 0.66 & 0.63 & $0.58^{\mathrm{a}}$ \\
\hline Work function $(\mathrm{eV})$ & \multicolumn{4}{|c|}{5.70} & $5.35^{\mathrm{b}}$ \\
\hline Surface energy $\left(\mathrm{J} / \mathrm{m}^{2}\right)$ & \multicolumn{4}{|c|}{2.69} & $2.45^{\mathrm{c}}$ \\
\hline
\end{tabular}

${ }^{a}$ FLAPW, seven-layer slab (Ref. 44).

${ }^{b}$ Experiment (Ref. 39).

${ }^{\mathrm{c}}$ Experiment (surface independent) (Ref. 40). 
In Fig. 1 it may be seen that the spin-polarization has little effect on the calculated work function, in contrast to, e.g., the case of iron. This was also found experimentally by Saito, ${ }^{45}$ who estimated the magnetic effect on the work function of $\mathrm{Ni}(001)$ to be less than $3 \mathrm{meV}$. Also, the surface energy of the $\mathrm{Ni}$ surfaces is very little affected by the spin-polarization (Fig. 2), indicating that the surface magnetism here is less stable than that of $\mathrm{Fe}$ and Co.

\section{SUMMARY}

We have implemented a spin-polarized version of our TB-LMTO Green's-function technique for surfaces and interfaces. The technique makes use of the frozen-core and atomic-sphere approximations, employs a minimal basis set, and uses the Green's-function approach to properly account for the breakdown of translational symmetry that occurs at the surface. We have applied this calculational scheme in a systematic study of magnetic moments, orbital compositions, work functions, and surface energies for the most closely packed ferromagnetic surfaces of iron, cobalt, and nickel. It is gratifying to see that in those cases where full-potential results exist we do find quantitative agreement with the moments and work functions obtained in these more accurate calculations. As to the magnetic surface energies, they are presented here and found to be within $10 \%$ of the values recommended by de Boer et al. ${ }^{40}$

In agreement with our earlier surface calculations for the alkali metals, we find that the calculated work function depends strongly on the choice of exchangecorrelation potential. The effect in $\mathrm{Fe}, \mathrm{Co}$, and $\mathrm{Ni}$ is close to $0.4 \mathrm{eV}$ and of the same size as the lowering of the work function brought about by the spin-polarization. This result does not seem to be appreciated in the literature and should be kept in mind when comparing with other calculations.

In comparison with experiment, we find that the calculations that employ the Vosko-Wilk-Nusair spinpolarized parametrization of the Ceperley-Alder manybody data systematically overestimate the work functions by $10 \%$, and that the von Barth-Hedin potential gives even higher work functions. We note that some discrepancy with experimental results is to be expected, since the work function of a rough surface is generally lower than that of a flat surface. ${ }^{47}$ Thus, the measured values tend to be lower bounds on the work function of the perfect surface. In this light we find the agreement with experiments highly satisfactory.

Our calculated work functions are also somewhat larger than those obtained by the FLAPW slab calculations. However, a recent full-potential Green's-function calculation for $\mathrm{Ni}(001)$ gave a work function similar to ours. We are therefore tempted to conclude that interslab interactions prevent the Fermi level from reaching the bulk value in the center of the slab, and hence might be responsible for the low work functions found in FLAPW calculations when compared to results obtained by Green's-function methods, which obey correct boundary conditions on both sides of the interface.

It is clear that the full-potential all-electron slab methods do carry high accuracy in representing the charge density, enabling proper studies of, e.g., the magnetic hyperfine field and valence electron properties of loosely packed surfaces, but we believe that the work function is less well represented. However, these earlier slab studies have enabled us to show that for the close-packed surfaces, the ASA, and the frozen-core approximation work well as regards the valence charge density and the spin density. In facing the general case of more open surface structures we expect that the ASA might be less appropriate and the extension to the full potential will then be necessary.

\section{ACKNOWLEDGMENTS}

M.A., S.M., and B.J. are grateful to The Göran Gustafsson Foundation and The Swedish Natural Science Research Council for financial support. Lars Nordström is acknowledged for stimulating and helpful discussions on the completion of this work. The work of H.L.S. and N.M.R. was supported by grants from the Novo Foundation, the Danish Natural Science Foundation (SNF), and the Danish Technical Science Foundation (STVF).
${ }^{1}$ Magnetic Properties of Low-Dimensional Systems edited by L.M. Falicov and J.L. Morán-López (Springer-Verlag, Berlin, 1986).

${ }^{2}$ C.S. Wang and A.J. Freeman, Phys. Rev. B 24, 4364 (1981).

${ }^{3}$ E. Wimmer, A.J. Freeman, and H. Kraukauer, Phys. Rev. B 30, 3113 (1984).

${ }^{4}$ O. Eriksson, G.W. Fernando, and R.C. Albers, Solid State Commun. 78, 801 (1991).

${ }^{5}$ G. Allan, Surf. Sci. Rep. 1, 121 (1981). See also A. Zangwill, Physics at Surfaces (Cambridge University Press, Cambridge, England, 1988).

${ }^{6}$ S. Ohnishi and A.J. Freeman, Phys. Rev. B 28, 6741 (1983).

${ }^{7}$ L.E. Klebanoff, S.W. Robey, G. Liu, and D.A. Shirley, Phys. Rev. B 30, 1048 (1984).

${ }^{8}$ C.L. Fu and A.J. Freeman, Phys. Rev. B 33, 1755 (1986).
${ }^{9}$ L. Liebermann, J. Clinton, D.M. Edwards, and J. Mathon, Phys. Rev. Lett. 25, 232 (1970).

${ }^{10}$ M. Landolt and M. Campagna, Phys. Rev. Lett. 38, 663 (1977).

${ }^{11}$ J.W. Krewer and R. Feder, Physica B 172, 135 (1991).

${ }^{12}$ E. Wimmer, H. Krakauer, M. Weinert, and A.J. Freeman, Phys. Rev. B 24, 864 (1981).

${ }^{13}$ C.-Q. Ma, M.V. Ramana, B.R. Cooper, and H. Krakauer, Phys. Rev. B 34, 3854 (1986).

${ }^{14}$ C.S. Wang and A.J. Freeman, Phys. Rev. B 21, 4585 (1980).

${ }^{15}$ O. Jepsen, J. Madsen, and O.K. Andersen, Phys. Rev. B 26, 2790 (1982).

${ }^{16}$ A.R. Williams, P.J. Feibelman, and N.D. Lang, Phys. Rev. B 26, 5433 (1982).

${ }^{17}$ P.J. Feibelman, Phys. Rev. B 35, 2626 (1987). 
${ }^{18}$ J.E. Inglesfield and G.A. Benesh, Phys. Rev. B 37, 6682 (1988).

${ }^{19}$ H.L. Skriver and N.M. Rosengaard, Phys. Rev. B 43, 9538 (1991).

${ }^{20}$ M. Scheffler, Ch. Droste, A. Fleszar, F. Máca, G. Wachutka, and G. Barzel , Physica B 172, 143 (1991).

${ }^{21}$ S. Crampin, K. Hampel, D.D. Vvedensky, and J.M. MacLaren, J. Mater. Res. 5, 2107 (1990).

${ }^{22} \mathrm{C}$. Li and A.J. Freeman, J. Magn. Magn. Mater. 75, 53 (1988).

${ }^{23}$ R. Zeller, P. Lang, B. Drittler, and P.H. Dederichs (unpublished).

${ }^{24}$ U. von Barth and L. Hedin, J. Phys. C 5, 1629 (1972).

${ }^{25}$ D.M. Ceperley and B.J. Alder, Phys. Rev. Lett. 45, 566 (1980).

${ }^{26}$ S.H. Vosko, L. Wilk, and M. Nusair, Can. J. Phys. 58, 1200 (1980).

${ }^{27}$ J.P. Perdew and A. Zunger, Phys. Rev. B 23, 5048 (1980).

${ }^{28}$ J.M. MacLaren, D.P. Clougherty, and R.C. Albers, Phys. Rev. B 42, 3205 (1990).

${ }^{29}$ O.K. Andersen, Phys. Rev. B 12, 3060 (1975)

${ }^{30}$ O. Gunnarsson, O. Jepsen, and O.K. Andersen, Phys. Rev. B 27, 7144 (1983)

${ }^{31}$ H.L. Skriver, The LMTO Method (Springer-Verlag, Berlin, 1984).

${ }^{32}$ O.K. Andersen and O. Jepsen, Phys. Rev. Lett. 53, 2571 (1984).
${ }^{33}$ O.K. Andersen, O. Jepsen, and D. Glötzel, in Highlights of Condensed-Matter Theory, edited by F. Bassani, F. Fumi, and M.P. Tosi (North-Holland, New York, 1985).

${ }^{34}$ O.K. Andersen, Z. Pawlowska, and O. Jepsen, Phys. Rev. B 34, 5253 (1986).

${ }^{35}$ W.R.L. Lambrecht and O.K. Andersen, Surf. Sci. 178, 256 (1986).

${ }^{36}$ P.O. Löwdin, J. Chem. Phys. 19, 1396 (1951).

${ }^{37}$ S.L. Cunningham, Phys. Rev. B 10, 4988 (1983).

${ }^{38}$ A.M. Turner, Y.J. Chang, and J.L. Erskine, Phys. Rev. Lett. 48, 348 (1982).

${ }^{39}$ H.B. Michaelson, J. Appl. Phys. 48, 4729 (1977).

${ }^{40}$ F.R. de Boer, R. Boom, W.C.M. Mattens, A.R. Miedema, and A.K. Niessen, in Cohesion in Metals, edited by F.R. de Boer and D.G. Pettifor (North-Holland, Amsterdam, 1988), Vol. I, p. 676.

${ }^{41}$ A.R. Miedema and B.E. Nieuwenhuys, Surf. Sci. 104, 491 (1981).

${ }^{42}$ C.L. Fu and A.J. Freeman, J. Magn. Magn. Mater. 69, L1 (1987).

${ }^{43}$ M.P. Pascal, C. R. Acad. Sci. Ser. C 264, 1270 (1967).

${ }^{44}$ C.L. Fu and A.J. Freeman, J. Phys. (Paris) 49, C8-1625 (1988).

${ }^{45}$ S. Saito, Jpn. J. Appl. Phys. 26, 1838 (1987).

${ }^{46}$ J.I. Lee, C.L. Fu, and A.J. Freeman, J. Magn. Magn. Mater. 62, 93 (1986).

${ }^{47}$ K. Besocke and H. Wagner, Phys. Rev. B 8, 4597 (1973). 